\title{
Form Perception of Partly Occluded Shapes in 4-Month-Old Infants
}

\author{
Tessa C. J. de Wit \\ Psychiatry Department \\ Radboud University Nijmegen Medical Centre \\ Karakter Child and Adolescent Psychiatry University Centre Nijmegen \\ Sven Vrins \\ Nijmegen Institute for Cognition and Information \\ Radboud University Nijmegen \\ Peter J. N. Dejonckheere \\ Department of Developmental, Personality, and Social Psychology \\ Ghent University \\ Rob van Lier \\ Nijmegen Institute for Cognition and Information \\ Radboud University Nijmegen
}

Two habituation experiments were conducted to investigate how 4-month-old infants perceive partly occluded shapes. In the first experiment, we presented a simple, partly occluded shape to the infants until habituation was reached. Then we showed either a probable completion (one that would be predicted on the basis of both local and global cues) or an improbable completion. Longer looking times were found for the improbably completed shape (compared to probable and control conditions), suggesting that the probable shape was perceived during partial occlusion. In the second experiment, infants were habituated to more ambiguous partly occluded shapes, where local and global cues would result in different completions. For adults, the per-

Correspondence should be addressed to Tessa C. J. de Wit. Psychiatry Department, Neuro Sensoric Cluster, Radboud University Nijmegen Medical Centre, P.O. Box 9101, 6500 HB Nijmegen, The Netherlands. E-mail: t.dewit@psy.umen.nl 
cept of these shapes is usually dominated by global influences. However, after habituation the infants looked longer at the globally completed shapes. These results suggest that by the age of 4 months, infants are able to infer the perceptual completion of partly occluded shapes, but for more ambiguous shapes, this completion seems to be dominated by local influences.

Most objects in our visual world undergo periods of partial occlusion, but we perceive a world that is composed of whole objects, not parts. The process that gives rise to these visual percepts, so-called visual completion, is effortless and is something of which we are not even aware. Note that this kind of completion is often referred to as amodal completion, but here we simply refer to it as completion. Take for example, the partly occluded shape in Figure 1a. Most individuals would judge the left shape to be a full rectangle (Figure 1b), not a mosaic shape (as in Figure 1c). Results obtained from different paradigms indicate that the generation of completions for simple, partly occluded shapes in adults takes about $250 \mathrm{msec}$ (Sekuler \& Palmer, 1992), and that different factors are important in the formation of completions, such as texture of the occluded region (Yin, Kellman, \& Shipley, 1997), size of the partly occluded region and the time for completion (Guttman, Sekuler, \& Kellman, 2003), relatability (Kellman \& Shipley, 1991), and simplicity (e.g., Van Lier, Van der Helm, \& Leeuwenberg, 1995).

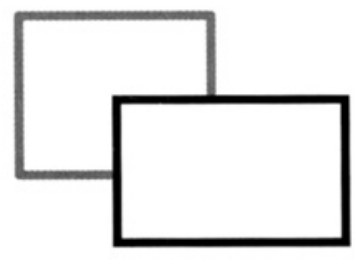

A)
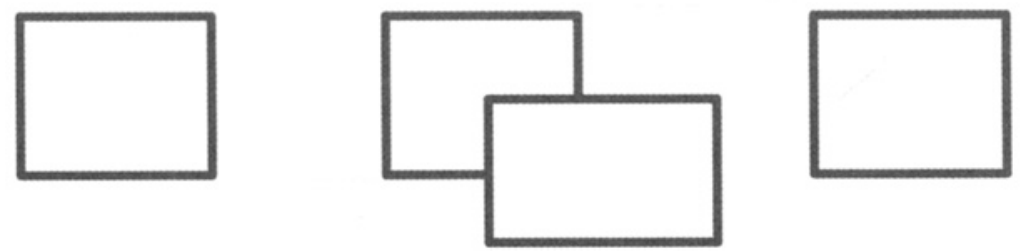

B)

C)

D)

FIGURE 1 (a) A partly occluded shape. (b) A completed form of the left shape. (c) A mosaic version of the left shape. (d) An anomalous completed form of the left shape. 
Infants already show feature binding at a young age (M. H. Johnson \& Mareschal, 2001; S. P. Johnson, 2000). That is, when objects that are partly hidden so that only two nonconnected parts are visible, they can perceive these two parts as belonging to one and the same object. In one of the first studies on perception of moving partly occluded shapes at a young age, Kellman and Spelke (1983) investigated the presence of the completion process in 4-month-old infants. Using a habituation paradigm, infants were shown a rod-and-box display (Figure 2a), with a box in front of a rod until they were habituated to this display, which is reflected in a decrease in looking time. Then, either a complete rod or two rods (i.e., a broken rod) were shown. Kellman and Spelke found that infants looked longer at the two rods, suggesting that the complete rod was most similar to what was perceived during habituation. Even at the age of 2 months, infants have already been found to display completion (S. P. Johnson \& Aslin, 1995).

Much research has focused on conditions needed to generate completion in infants: Besides motion, stationary information sources such as alignment, curvature, and good form of the occluded shape have also been found to be important for the formation of completion. For example, S. P. Johnson, Bremner, Slater, and Mason (2000) looked at the role of good form (an overall regular shape; see, e.g., Figure $2 b$ ) and curvature (e.g., Figure $2 c$ ) in the generation of completion processes for 4-month-old infants, by showing partly occluded crosses (Figure 2b). The lines of the crosses going behind the occluder were not aligned (as opposed to the bars in

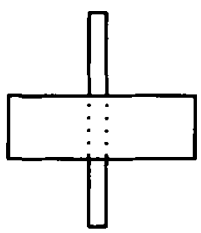

A)

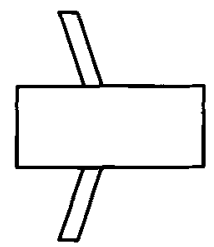

D]

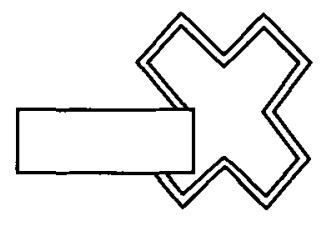

B)

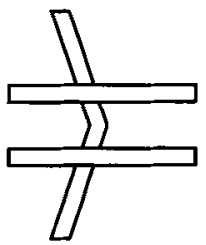

E)

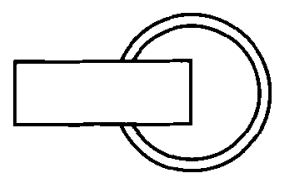

C)

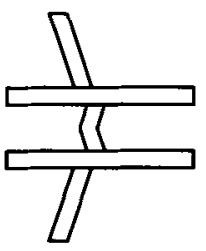

F)

FIGURE 2 (a) A rod-and-box display. (b) A partly occluded cross (after S. P. Johnson et al., 2000). (c) A partly occluded circle (after S. P. Johnson et al., 2000). (d) A partly occluded shape, which could either be completed so that it is (e) orientation consistent, or (f) orientation inconsistent (after S. P. Johnson et al., 2002). 
Figure 2a), but still infants perceived partly occluded crosses as whole objects. This demonstrated that, for an overall regular shape, lines do not have to be aligned to generate completion in infants.

Not only do adults know that objects continue, not end, when they disappear behind another object, but more important, we make specific inferences about the shape of a partly occluded object. For example, we would be surprised if the left shape of Figure la turned out to be a complete shape as in Figure ld. We would consider this type of completion to be improbable. In other words, we perceive a certain form of the part of the object that it is partly hidden behind another object. In a study that directly addressed the issue of the nature of completions in infants, Craton (1996) investigated the percept of a stationary vertical rectangle that was partly occluded in the middle. When the occluder was removed either a complete rectangle became visible, or a horizontal part became visible, rendering a cross. Only 8-month-olds, but not 5.5-month-olds and 7-month-olds, differentiated between the full rectangle and the full cross. S. P. Johnson, Bremner, Slater, Mason, and Foster (2002) investigated whether infants could differentiate between a completion in which the orientation is consistent or inconsistent with the already visible parts of a partly occluded shape. After having seen the partly occluded shape of Figure 2d, 7-month-old infants, but not 4-month-olds, could differentiate between the orientation-consistent (Figure $2 \mathrm{e}$ ) and orientation-inconsistent completion (Figure 2f).

The study by S. P. Johnson et al. (2002) shows that the age at which infants can perceive object completion can be earlier than the 8 months concluded from the Craton (1996) study. A major difference between these studies lies in the absence and presence of motion of the partly occluded shape. In the S. P. Johnson et al. (2002) study, the partly occluded object moved back and forth. Object motion is well known to provide the earliest source of information on object unity during development (S. P. Johnson \& Aslin, 1996). However, the test displays used in the S. P. Johnson et al. (2002) study might still restrict the age at which infants can perceive the specific form of a completion. That is, the finding that 4-month-olds do not differentiate between probable and improbable shapes might be due to the complexity of the test displays. In their study, the habituation phase consisted of two rod parts that were misaligned, but relatable across the occluder. The middle part of the occluder was removed in the test phase, and a third rod part was visible (Figures $2 \mathrm{e}, 2 \mathrm{f}$ ). The test displays were therefore quite complex: The completion (the third rod part) was still not directly connected to the two rods that were initially visible. Thus, infants would still have to complete the three separate rod parts behind the occluder. Supplying more visual information about the resulting form (i.e., the complete shape) could increase the sensitivity of the method.

If 4-month-olds indeed perceive a specific form during partial occlusion, the question then is what cues would determine this perceived form. This becomes clear if we consider a partly occluded object that is more ambiguous with regard to 
the form of the hidden part. For example, for the partly occluded shape in Figure $3 a$, one can think of different kinds of completions. Two main influences have been proposed that, depending on the nature of the partly occluded shape, result in different completions for adults. Local influences, on the one hand, are based on information that is available at the contour intersections (see the arrows in Figure $3 \mathrm{a}$ ) between the partly occluded shape and the occluder, and result in smooth completions. As described by the Gestalt principle of good continuation, the contours are linearly extended behind the occluder. Figure $3 \mathrm{~b}$ shows the result of local influences. Global influences, on the other hand, result from integrating all information from the visible part of the partly occluded shape to form a completion, so that the completion adds up to an overall regularity of the partly occluded shape despite local irregularities. This resulting shape is described by the Gestalt psychologists as being a "good form," which is figurally the "simplest" solution (Koffka, 1935). Global factors include stimulus aspects such as symmetry and iteration, where symmetrical contours or contours containing many repeating elements result in overall regular shapes (e.g., Van Lier, Van der Helm, \& Leeuwenberg, 1995). Figure 3c shows a global completion, which has actually been shown to be the dominating completion for these kinds of stimuli in adults (De Wit, Mol, \& Van Lier, 2005; De Wit \& Van Lier, 2002). In a magnetoencephalography (MEG) study it was shown that there is an early left occipital component that is mainly sensitive to purely figural aspects from the stimulus itself, but that it is already modulated by the perceived completion, which is mainly based on global influences (De Wit, Bauer, Oostenveld, Fries, \& Van Lier, 2006). It is now generally agreed that whether local or global influences dominate in the completion process depends on the nature of the stimuli. For example, global influences dominate completion for regular shapes (Sekuler, 1994; Van Lier, Leeuwenberg, \& Van der Helm, 1995; Van Lier, Van der Helm, \& Leeuwenberg, 1995; Van Lier \& Wagemans, 1999). However, although we also found global influences to be dominant in younger individuals (in a study in which the focus was on autism), the control group ( $M$ age $=11$ years) did show a tendency for an in-

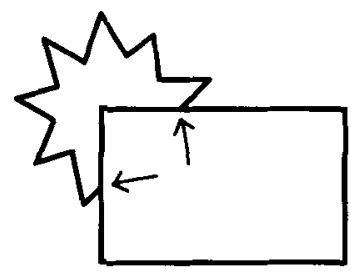

A)

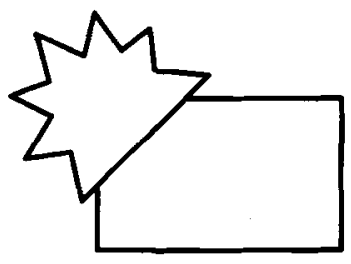

B)

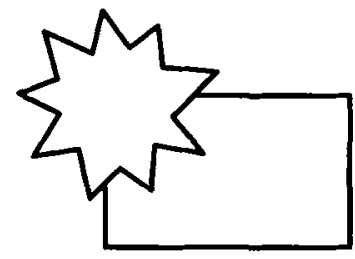

C)

FIGURE 3 (a) A partly occluded shape. (b) A locally completed form of the left shape. (c) A globally completed form of the left shape. 
creased effect of local influences compared to adults for the sorts of shapes shown in Figure 3 (De Wit, Schlooz, Hulstijn, \& Van Lier, 2007).

S. P. Johnson et al. (2000) provided evidence for the beneficial effects of global information in completion in 4-month-olds. The presence of "good form" in the partly occluded shapes determined whether or not these infants perceived a completed shape. However, their study did not address how well-defined the perceived shape of the completion was. Therefore in Experiment 1, we first turn to the question of how well defined form perception in completion is for 4-month-old infants. This was tested with shapes in which both local and global influences would result in the same completion, and this completion is compared to one that is more improbable (as in Figure 1). Furthermore, as the role of these local and global influences for 4-month-old infants is not clear, we investigated them under conflicting conditions in infants in Experiment 2. That is, for these infants we examined form perception in completion for partly occluded shapes, in which the form of the completion is more ambiguous due to the figural context (as in Figure 3).

\section{EXPERIMENT 1}

To investigate whether 4-month-olds do have well-defined percepts of the form of a partly occluded shape, we used test displays that provide the infants with more information regarding the complete shape. Infants were first habituated to a moving, partly occluded, simple shape. This partly occluded shape was kept simple (see Figure 4a) to minimize the ambiguity of the completion. That is, based on both local and global factors, a completion would be expected that forms a rectangle (Figure $4 \mathrm{c}$ ). Where the local factors result in a linear continuation of the contours behind the occluder, the global factors result in an overall symmetrical pattern, which for this shape is also the linear continuation. The full square is thus considered to be a probable completion. In comparison to this, the completion shown in Figure $4 \mathrm{~d}$ is more complex and the overall shape does not conform to the principles of linear continuation and overall symmetry, and is therefore defined as an improbable completion. After habituation, we showed the probable completion and the improbable completion on alternating test trials. Given the tendency of young infants to prefer novel stimuli relative to familiar stimuli after habituation, the time the infant looks at the test display will be relatively short when this test display is perceived to be similar to the habituation display and longer if the infant considers the test display to be novel. To control for any intrinsic preference for one of the test displays, another group of infants was run in a control condition, in which they were habituated to an orange triangle (thus unrelated to the shapes in the test phase). We hypothesized that 4-month-olds will perceive the (im)probability of one completion and will thus look longer at the improbably completed shape only in the experimental condition. 


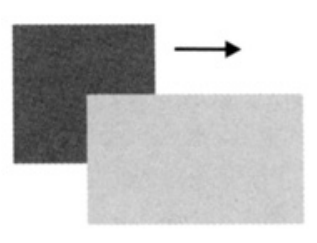

A)

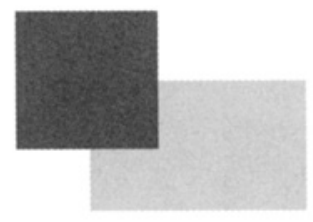

C)

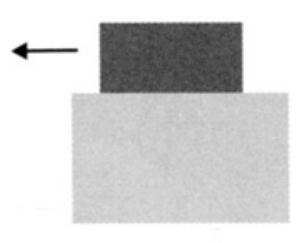

B)

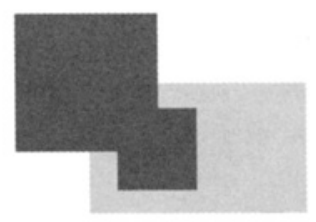

D)

FIGURE 4 (a) The partly occluded shape used in Experiment 1, in the leftmost position. (b) The partly occluded shape used in Experiment 1, in the rightmost position. (c) The probable completion of the left shape. (d) The improbable completion of the left shape.

\section{Method}

Participants. The final sample in the experimental group consisted of 14 infants ( 7 girls; $M$ age $=129.3$ days, $S D=9.7$ ); the control group consisted of 12 infants ( 7 girls; $M$ age $=130.8$ days, $S D=13.6$ ). Seven more infants were observed but excluded from the analyses because of fussiness $(n=3)$ or persistent inattention toward the displays $(n=2)$. Names and addresses of the participants were obtained from the municipal government in Ghent, Belgium, and participants were recruited by letter. After the experiment, the infants were offered a toy; parents were not compensated for participation.

Apparatus and stimuli. A Macintosh Powerbook G4 and a video projector were used to control the experiment and generate the stimuli. Stimulus presentation and data collection were controlled by custom habituation software (Cohen, Atkinson, \& Chaput, 2004). There were two experimenters: one performing online coding and one recording with video cameras for later analyses by a second observer, blind to the stimuli and experimental hypotheses. An observer who was blind to the stimulus that was presented to the infant recorded looking times by pressing a key as the infant looked at the stimulus or by releasing the key as the in- 
fant looked away. The computer presented the stimulus displays, stored the observer's data, calculated the habituation criterion for each infant, and changed displays after the habituation criterion was met. Sessions were recorded by two digital video cameras (Panasonic AW-E300E and Sony DSR-PD150P). One camera was mounted on the ceiling behind the infant, registering the stimulus presentation. The other camera had been placed before a small gap inside the panel in front of the infant and registered the infant's gaze. Both video signals were transmitted to a mixing console, combined, and recorded on video. This new video signal was in turn transmitted to a monitor near the experimenter. On the left side of the monitor screen the stimulus movements were presented, and on the right side the infant could be seen. Cardboard was positioned in the middle of the monitor screen, obscuring the view of the display box for the experimenter who measured the infant's looking behavior. Consequently, this experimenter was blind to what was happening in the display box and did not know the order in which the events were presented. Observers were blind to the stimuli with which infants were presented.

The habituation (occlusion) display consisted of a $15.2 \times 15.2 \mathrm{~cm}$ moving blue square, subtending $8.7^{\circ} \times 8.7^{\circ}$ visual angle, that was partly occluded by a stationary green rectangle, $23.4 \times 14.2 \mathrm{~cm}$ and $13.4^{\circ} \times 8.1^{\circ}$. Only the blue square moved in a lateral way at a rate of $4.6 \mathrm{~cm} / \mathrm{sec}$, changing direction every $2.5 \mathrm{sec}$ : Figure $4 \mathrm{a}$ shows both the leftmost and Figure $4 \mathrm{~b}$ the rightmost position of the moving blue square. The square and the rectangle were presented against a black rectangular background, filled with a grid of $12 \times 20$ white dots, measuring $48.2 \times 34.7 \mathrm{~cm}$ and $27.1^{\circ} \times 19.7^{\circ}$. The control habituation display consisted of a $8.6^{\circ} \times 8.6^{\circ} \mathrm{cm}$ orange triangle (pointing upward) that also moved in a lateral way at a rate of $4.6 \mathrm{~cm} / \mathrm{sec}$, changing direction every $2.5 \mathrm{sec}$. Test displays were similar to the habituation displays, only now the moving blue rectangle was in front of the green rectangle, revealing the occluded part that was completed as a regular square (probable completion, Figure 4c), or extended with an extra part (improbable completion, Figure $4 d)$. The blue rectangle again moved in a similar fashion as in the habituation display. The attention-getter consisted of a green circle that expanded and contracted, accompanied by a dong sound.

Procedure. Each infant was seated on a parent's lap, positioned about 100 $\mathrm{cm}$ from the display. Parents were told not to interact with their infants. At the beginning of each trial an attention-getter was presented, followed by the habituation display. Each trial was presented until the infant looked away for more than $2 \mathrm{sec}$ or had looked at the display for $60 \mathrm{sec}$ (the maximum trial length). The habituation phase was ended when the habituation criterion was met, which was defined as a decrease in total looking time during four consecutive trials, resulting in less than half of the looking time on the first four trials. When the habituation criterion was met, the computer switched to the test phase. Here, the same shape was shown in front of the occluder, with either a probable or improbable completion. The two 
test displays (one block) were each shown three times in alternation and the order of presentation was balanced. To keep the infants involved, attention-getters were presented in between all trials.

\section{Results and Discussion}

Interobserver agreement was high ( $M$ Pearson $r=.98$ ). As the data were skewed, the data were log-transformed (the raw data are shown in the graphs).

Habituation phase. The mean number of habituation trials necessary to reach the habituation criterion was 7.9 trials for the experimental condition and 8.8 trials for the control condition. An independent samples $t$ test showed that this was not significant, $t(24)=0.812, p=.425$, effect size $r=.16$. An independent samples $t$ test was performed for total looking time in the two habituation conditions (experimental vs. control), which was also not significant, $t(24)=0.384, p=.704$, $r=.08$.

Test phase. Looking times averaged over the three blocks are plotted in Figure 5. A mixed-model analysis of variance (ANOVA) was performed for looking time with block (3: first, second, and third pair of trials, to check for general decrease in looking time) and probability (2: probable and improbable completion) as within-subjects factors, and condition (2: experimental or control) and order ( 2 : probable or improbable first) as between-subject factors. This revealed a signifi-

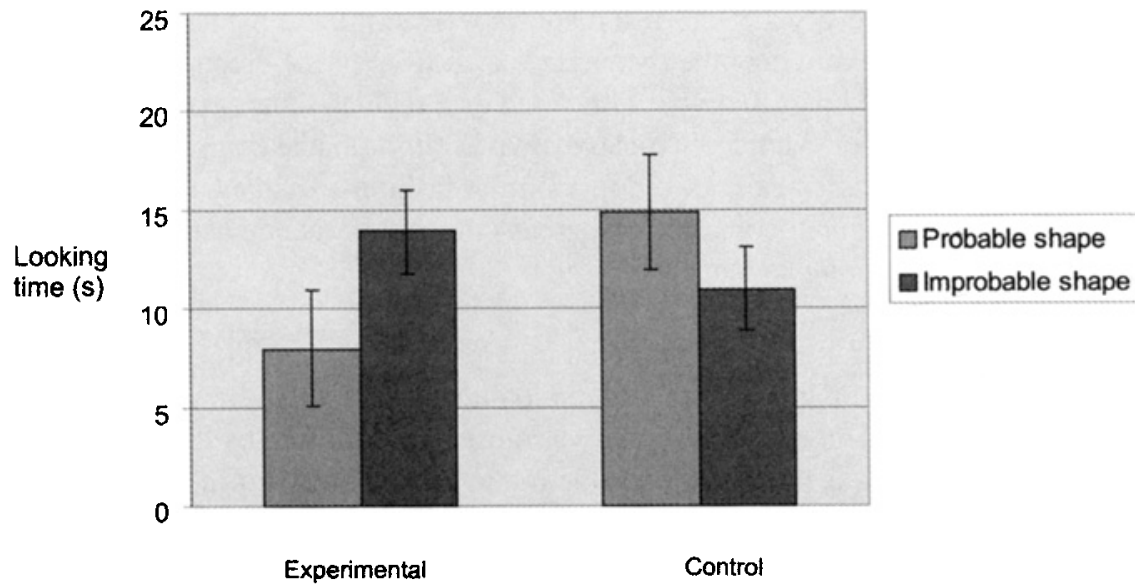

Condition

FIGURE 5 Looking times (in sec) for probable and improbable shapes in the experimental and the control condition, averaged over the three blocks. Error bars represent standard error of the mean. 
cant main effect for block, $F(2,21)=6.49, p<.01$, due to an overall decline in looking time across trials, and a significant iBlock $\times$ Condition $\times$ Order interaction, $F(2,21)=3.64, p<.05$, which stemmed from the longer looking times in the first block for the infants in the control condition and in the experimental improbable condition. Finally, there was a Probability $\times$ Condition interaction, $F(1,22)=$ $7.17, p<.05$. Post hoc analyses (simple effects tests) revealed no significant preference for one of the displays in the control condition, $F(1,11)=1.52, p=.24, r=$ .35 , indicating that the difference in looking time in the experimental condition was not due to a higher attractiveness of the improbable shape per se. In the experimental condition, infants showed longer looking times for improbable shapes as compared to probable shapes, $F(1,13)=8.02, p<.05, r=.62$. Comparing the two groups for the shapes revealed no difference between the groups for the improbable completion, $F(1,24)=0.13, p=.72, r=.07$, but a significant difference between the groups for the probable completion, with shorter looking in the experimental condition, $F(1,24)=5.79, p<.05, r=.44$.

The results show that when first habituated to a partly occluded shape, infants afterward indeed differentiated between the probable and improbable shape. As expected, they looked less at the probable shape compared to the improbable shape. This preference was not apparent in the control condition; infants did not have a significant preference for either shape. We therefore conclude that at the age of 4 months, infants already have a fairly clear percept of a moving, partly occluded shape in terms of form of the occluded region.

\section{EXPERIMENT 2}

What happens if a partly occluded object is more ambiguous with regard to the form of the hidden part? In this experiment, infants were habituated to a partly occluded shape that could lead to qualitatively different completions, resulting from either local or global influences (see Figure 6). As mentioned earlier, for these shapes, the global influences are dominant in adults (De Wit \& Van Lier, 2002). If global influences indeed already dominate (as suggested by S. P. Johnson et al., 2000) in completion for infants, we expect infants to look longer at the locally completed shapes.

\section{Method}

The methods are identical to those in Experiment 1, unless stated otherwise.

Participants. The final sample in the experimental group consisted of 20 infants ( 6 girls; $M$ age $=120.5$ days, $S D=5.3$ ); the control group consisted of 19 infants ( 4 girls, $M$ age $=119.6$ days, $S D=8.1$ ). Two more infants were observed but excluded from the analyses because of persistent inattention toward the displays. 


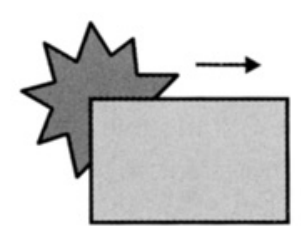

A)

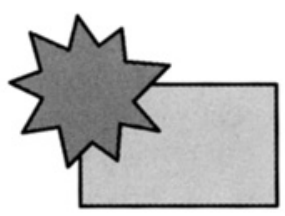

C)

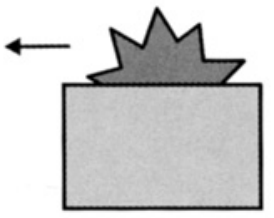

B)

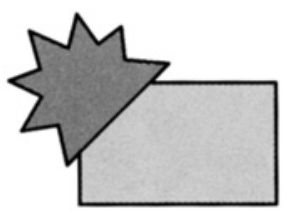

D)

FIGURE 6 (a) The partly occluded shape used in Experiment 2, in the leftmost position. (b) The partly occluded shape used in Experiment 2, in the rightmost position. (c) The global completion of the left shape. (d) The local completion of the left shape.

Apparatus and stimuli. The habituation (occlusion) display consisted of a $18.8 \times 17.8 \mathrm{~cm}$ blue, starlike shape, subtending $10.7^{\circ} \times 10.2^{\circ}$ visual angle that was partly occluded by the green rectangle, and again moved in a lateral way at a rate of $4.6 \mathrm{~cm} / \mathrm{sec}$, changing direction every $2.5 \mathrm{sec}$. Figure 6 a shows the leftmost and Figure $6 \mathrm{~b}$ the rightmost position of the starlike shape. The starlike figure and the rectangle were presented against the black regular background, filled with a grid of 12 $\times 20$ white dots. The control habituation display was identical to the one used in Experiment 1 . Test displays were similar to the habituation displays, only now the starlike shape was in front of the blue box, where the occluded part was completed as a regular starlike shape (global completion, Figure $6 c$ ), or the contour was linearly continued (local completion, Figure 6d).

\section{Results and Discussion}

Interobserver agreement again was high (mean Pearson $r=.99$ ).

Habituation phase. The mean number of habituation trials necessary to reach the habituation criterion was 10.1 trials for the experimental condition and 9.9 trials for the control condition. An independent samples $t$ test showed that this was not a significant difference, $t(37)=0.195, p=.847, r=.03$. An independent samples $t$ test performed for total looking time in the two conditions (experimental vs. control) was also not significant, $t(37)=0.395, p=.695, r=.06$. 
Test phase. Looking times averaged over the three blocks are plotted in Figure 7. A mixed-model ANOVA was performed for looking time with block (3: first, second, and third pair of trials) and globality (2: global and local completion) as within-subjects factors, and condition (2: experimental or control) and order (2: global or local first) as between-subject factors. This revealed a significant main effect for block, $F(2,34)=4.76, p<.05$, due to an overall decline in looking time across trials and a Block $\times$ Globality $\times$ Condition interaction, $F(2,34)=8.93, p<$ .005 . This stemmed from the longer looking times in the first block for the infants in the control condition for both the local and global shapes, and in the experimental condition this longer looking time was there only for the global shape. Finally, there was a significant Globality $\times$ Condition interaction, $F(1,35)=4.27, p<.05$. Post hoc analyses (simple effects tests) revealed no significant preference for one of the displays in the control condition, $F(1,18)=0.50, p=.49, r=.16$, indicating that the difference in looking time in the experimental condition was not due to a higher attractiveness of one of the shapes per se. In the experimental condition infants showed shorter looking times for local shapes as compared to global shapes, $F(1,19)=4.57, p<.05, r=.44$. Comparing the two groups for the shapes revealed no difference between the groups for the global completion, $F(1,37)=3.74, p=.06$, $r=.30$, but a significant difference between the groups for the local completion, with shorter looking in the experimental condition, $F(1,37)=9.93, p<.005, r=.46$.

The results show that when first habituated to a moving, partly occluded ambiguous shape, infants again differentiated between the different shapes. Whereas the

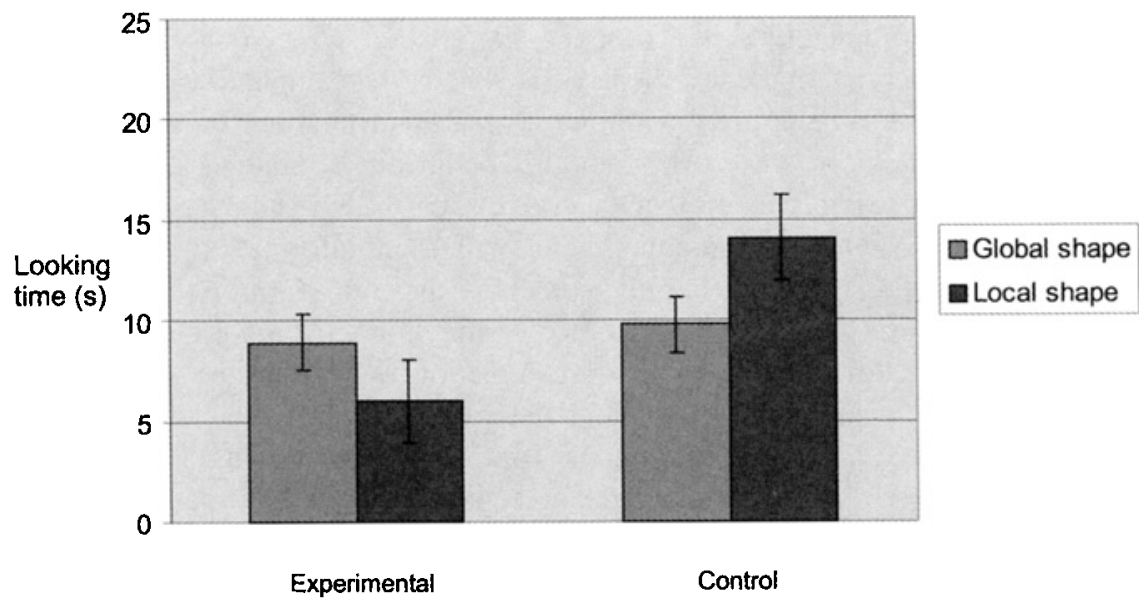

Condition

FIGURE 7 Looking times (in sec) for global and local shapes in the experimental and the control condition, averaged over the three blocks. Error bars represent standard error of the mean. 
infants do not differentiate between the local and global shapes in the control condition, they actually look less at the local shape in the experimental condition. We therefore conclude that local influences dominate the completion process at the age of 4 months. Infants thus perceive these shapes in a different way than adults and normally developing children around the age of 11 years (e.g., De Wit et al., 2007; De Wit \& Van Lier, 2002). This striking result might stem from the more ambiguous shape. Although our finding seems to conflict with those of S. P. Johnson et al. (2000) showing sensitivity to global cues, that particular study did not directly contrast the influence of global and local cues. The findings reported here on the dominance of local influences may also be related to the higher degree of complexity of the shape, in terms of symmetry (e.g., Van Lier, Van der Helm, \& Leeuwenberg, 1995). That is, the higher complexity might force the infants into more detailed information processing than would be activated by the simpler shape used in Experiment 1.

\section{GENERAL DISCUSSION AND CONCLUSIONS}

These two experiments show that 4-month-old infants already have a fairly clear perception of a moving, partly occluded shape, which is at an earlier age than reported before (Craton, 1996; S. P. Johnson et al., 2002). S. P. Johnson et al. showed that in displays in which the partly occluded shape moves, the age of infants capable of perceiving a clear form of the occluded part emerges around the age of 7 months. Our study thus shows that infants can already perceive the form of the occluded part of a simple object at a younger age. This difference in age can be explained by the fact that in the test displays used here, the complete shape is available to the infants in its entirety with only a small part missing. In addition, the surface of the structure that has to be continued behind the occluder is larger for the shapes used in these experiments than the rods used in the S. P. Johnson et al. study. Both of these differences provide the infants with more information, thereby enabling the formation of a more definite percept of the entire form. As more information requires less ambiguity about visual completion, this might facilitate performance in our task. Ontogenetically, completion might thus be a gradually developing process and not an all-or-none process.

When presented with more ambiguous shapes in which local and global influences result in different completions, the percept of 4-month-old infants is dominated by local influences. Thus, there is a qualitative difference in the percept of these partly occluded shapes, as compared to adults and children with and without autism, where global influences have been found to dominate (De Wit et al., 2007; De Wit \& Van Lier, 2002). This finding on the developmental delay of global dominance agrees with findings by Porporino, Shore, Iarocci, and Burack (2004). Porporino reported that global factors, as compared to local factors, are still more 
vulnerable in form perception in 6- and 8-year-olds as compared to older children and adults. This study provides support for a qualitative difference in the development of the form percept resulting from completion. We speculate that the

reason 4-month-old infants show a more local type of processing might be due to the degree of control of eye movements (e.g., Von Hofsten, 2003). The integration of information that is needed for global completion might thus require extra cues. All in all, this again shows the gradual nature of the development of completion; whether and how partly occluded shapes are perceived as wholes depends on the information that is available. The question remains at what age these global influences begin to play an influential role in perception.

\section{ACKNOWLEDGMENTS}

Tessa C. J. de Wit was funded by the Niels Stensen Foundation. We would like to thank all the children and their parents for their invaluable help.

\section{REFERENCES}

Cohen, L. B., Atkinson, D. J., \& Chaput, H. H. (2004). Habit X: A new program for obtaining and organizing data in infant perception and cognition studies (Version 1.0). Austin: University of Texas.

Craton, L. E. (1996). The development of perceptual completion abilities: Infants' perception of stationary, partly occluded objects. Child Development, 67, 890-904.

De Wit, T. C. J., Bauer, M., Oostenveld, R., Fries, P., \& Van Lier R. (2006). Magnetic brain responses to the interplay of local and global influences in visual completion. Neurolmage, 32, 1815-1825.

De Wit, T. C. J., Mol, K. R., \& Van Lier R. (2005). Investigating metrical and structural aspects of visual completion: Priming versus searching. Visual Cognition, 12, 409-428.

De Wit, T. C. J., Schlooz, W. A. J. M., Hulstijn, W., \& Van Lier R. (2007). Visual completion and complexity of visual shape in children with pervasive developmental disorder. European Child \& Adolescent Psychiatry, 16, 168-177.

De Wit, T. C. J., \& Van Lier R. J. (2002). Global visual completion of quasi-regular shapes. Perception, $31,969-984$.

Guttman, S. E., Sekuler, A. B., \& Kellman, P. J. (2003). Temporal variations in visual completion: A reflection of spatial limits? Journal of Experimental Psychology: Human Perception and Performance, 29, 1211-1227.

Johnson, M. H., \& Mareschal, D. (2001). Cognitive and perceptual development during infancy. Current Opinion in Neurobiology, 11, 213-218.

Johnson, S. P. (2000). The development of visual surface perception: Insights into the ontogeny of knowledge. In C. Rovee-Collier, L. Lipsitt, \& H. Hayne (Eds.). Progress in infancy research (Vol. 1, pp. 113-154). Hillsdale, NJ: Lawrence Erlbaum Associates, Inc.

Johnson, S. P., \& Aslin, R. N. (1995). Perception of object unity in 2-month-old infants. Developmental Psychology, 31, 739-745.

Johnson, S. P., \& Aslin, R. N. (1996). Perception of object unity in young infants: The roles of motion, depth, and orientation. Cognitive Development, 11, 161-180. 
Johnson, S. P., Bremner, J. G., Slater, A. M., \& Mason, U. C. (2000). The role of good form in young infants' perception of partly occluded objects. Journal of Experimental Child Psychology, 76, 1-25.

Johnson, S. P., Bremner, J. G., Slater, A. M., Mason, U. C., \& Foster, K. (2002). Young infants’ perception of unity and form in occlusion displays. Journal of Experimental Child Psychology, 81, 358-374.

Kellman, P. J., \& Shipley, T. F. (1991). A theory of visual interpolation in object perception. Cognitive Psychology, 23, 141-221.

Kellman, P. J., \& Spelke, E. S. (1983). Perception of partly occluded objects in infancy. Cognitive Psychology, 15, 483-524.

Koffka, K. (1935). Principles of Gestalt psychology. London: Routledge \& Kegan Paul.

Porporino, M., Shore, D. I., Iarocci, G., \& Burack, J. A. (2004). A developmental change in selective attention and global form perception. International Journal of Behavioral Development, 28, 358-364.

Sekuler, A. B. (1994). Local and global minima in visual completion: Effects of symmetry and orientation. Perception, 23, 529-545.

Sekuler, A. B., \& Palmer, S. E. (1992). Perception of partly occluded objects: A microgenetic analysis. Journal of Experimental Psychology: General, 121, 95-111.

Van Lier, R., Leeuwenberg, E., \& Van der Helm, P. (1995). Multiple completions primed by occlusion patterns. Perception, 24, 727-740.

Van Lier, R., Van der Helm, P., \& Leeuwenberg, E. (1995). Competing global and local completions in visual occlusion. Journal of Experimental Psychology: Human Perception and Performance, 21, 571-583.

Van Lier, R., \& Wagemans, J. (1999). From images to objects: Global and local completions of self-occluded parts. Joumal of Experimental Psychology: Human Perception and Performance, 25, 1721-1741.

Von Hofsten, C. (2003). On the development of perception and action. In J. Valsiner \& K. J. Connolly (Eds.), Handbook of developmental psychology (pp. 114-140). London: Sage publications.

Yin, C., Kellman, P. J., \& Shipley, T. F. (1997). Surface completion complements boundary interpolation in the visual integration of partly occluded objects. Perception, 26, 1459-1479. 